narrowly fluctuating tropics, where the highest speciation rates are likely ${ }^{2}$. Indeed, the diversity of species in the genus Drosophila in Australia is highest in the humid tropics, whereas at more widely fluctuating heat-stressed forest edges, species diversity is lower, corresponding to the depauperate faunas characteristic of the heat-stressed habitats of the wet-dry tropics?

In conclusion, and in agreement with the palaeontological evidence ${ }^{1.2}$, maximum speciation rates are expected in habitats characterized by narrowly fluctuating environments implying moderate stress, moderate genetic variability of ecologically important traits, and not unduly restrictive metabolic costs. In this way, gaps between palaeonto-

\section{Schroedinger's quantum cat}

SIR - Schroedinger's imaginary cat ${ }^{1-3}$, supposed to be in a linear combination of the quantum states live and dead, emphasizes the contrast between classical and quantum systems - and of the ambiguity of the boundary between them in the usual interpretation of measurement in quantum mechanics. Dissatisfaction with such ambiguity has led many physicists to construct alternative quantum theories. It may be held, of course, that the states of cats, which are macroscopic systems, are irrelevant to quantum measurement, but is that necessarily so?

Consider a molecule of DNA, which can be mutated genetically by the influence of photons. The orthodox view ${ }^{4}$ is that a quantum measurement is "any process of interaction between classical and quantum objects occurring apart from and independently of any observer". It is important to be clear about the distinction between the classical and quantum systems used in measurement. The states of classical systems are robust and can be preserved and reproduced, with only small probability of error, like the characters on this page or the state of survival of a real cat. They form a classical record.

By contrast, the reproducibility of quantum systems is constrained by the no-cloning theorem of Wooters and Zurek ${ }^{5}$ and Ghirardi and Weber ${ }^{6}$. The information in a set of quantum states can easily be destroyed.

It is usually assumed that the classical measuring apparatus must be physically macroscopic, but this is not the case for a molecule of DNA. Yet the sequence of base pairs is a classical record, cloning is the norm, parts of the record may be retained over millions of years and may be reproduced more often than any published record of a measurement. With the help of biological proof-reading, the error in copying DNA is estimated to be between $10^{-7}$ and $10^{-11}$ per replication of a base pair ${ }^{7}$.

But DNA also responds to quantum events, as when mutations are produced by single photons, with consequences that may be macroscopic - leukaemia, for example. logy, physiology, ecology and evolutionary genetics are being bridged with the level of physical stress, especially climatic, being the connecting environmental link.

P. A. Parsons

Department of Zoology,

University of Adelaide,

Adelaide, South Australia 5000,

Australia

1. Sheldon, P. R. Nature 330, 561-563 (1987).

2. Sheldon, P. R. Nature 345, 772 (1990).

3. Hoffmann, A. A. \& Parsons. P. A. Evolutionary Genetics and Environmental Stress (Clarendon, Oxford 1991)

4. Parsons, P. A. Trends Ecol. Evol. 5, 315-317 (1990)

5. Moore, P. D. Nature 306, 17 (1983).

6. Petraitis, P., Latham, R. E. \& Neisenbaum, R. F. Quart. Rev Biol. 64, 393-418 (1989).

Parsons, P. A. Proc. ecol. Soc. Aust. 13, 43-49 (1985)

Moreover, these consequences may be much delayed, as in recessive mutations, so that a classical DNA record may be kept at the molecular level for several generations before it is expressed macroscopically. Such a mutation is a classical record, on the molecular scale, of the quantum event that produced the mutating photon, so that it represents a quantum measurement. Thus quantum measurements do not require macroscopic systems. The total energy of the

\section{Whose larvae?}

SIR - The inability to distinguish between marine invertebrate larvae of closely related taxa is a long-standing problem in marine ecology. Within groups such as bivalves or barnacles, the larvae of many species are so similar that they cannot be identified by morphology alone'. Using the polymerase chain reaction to amplify and sequence mitochondrial DNA, we are able to distinguish between the morphologically identical, cooccurring larvae of two species of sea cucumber This technique should prove effective for the identification of larvae even when they are far from their adult population, such as open-ocean (teleplanic) larvae or the larvae of deep-sea organisms.

The sea cucumbers Psolus fabricii and Cucumaria frondosa are common members of the subtidal communities along the northeast coast of the United States. P. fabricii occurs primarily on hard surfaces, $C$. frondosa is found on both hard-rock and soft-sediment bottoms ${ }^{2,3}$. In 1990 , we con-

Psolus ACT-GAAaACAgatatagtTACCGCAG

Cucumaria C..G.T......A.A........

Psolus ggataAcagcgtcatctcctTtaAgag

Cucumaria ...................

Sequence of a portion of the $16 \mathrm{~S}$ rRNA gene from two species of sea cucumber in the Gulf of Maine. Individual larvae were placed directly into an amplification buffer containing primers to the conserved region of the gene. Sequences were determined directly from the amplified products using a nested pair of primers. bonds that bind a base pair can be less than the energy of the photon that produces the mutation, so that quantum measurements do not require amplification either.

Schroedinger chose his example well. Most people find it difficult or impossible to believe that an object of affection such as a cat could be in a state which is a linear superposition of a live state and a dead state. But if a classical record is what matters, a quantum superposition of viable and lethal sequence of base pairs in DNA is as absurd as a cat that is both alive and dead. Should one seek to learn more about Schroedinger's cat by studying her kittens?

School of Mathematical Sciences, Queen Mary and Westfield College, Mile End Road, London E1 4NS, UK

1. Schroedinger, E. Naturwissenschaften 23, $807-812$
(1935).
2. Schroedinger, E. Naturwissenschaften 23, $823-828$
(1935).
3. Schroedinger, E. Naturwissenschaften 23, 844-849
(1935).
4. Landau, L. \& Lifshitz, E. Quantum mechanics (Pergamon,
London, 1958).
5. Wooters, W. K. \& Zurek, W. H. Nature 299, 802-803
(1982).
6. Ghirardi, G. C. \& Weber, T. Nuovo Cim. 78B, 9-20 (1983).
7. Uatson, J. D. Ct al. in Molecular Biology of the Gene
(Cummings, Menlo Park, 1988). ducted plankton tows around the Isles of Shoals, New Hampshire, to collect larvae of $P$. fabricii. Bright red pentacula larvae were caught in large numbers in March, and then again in late May. Based on the advice of local embryologists, and on the similarity of the larvae to those of the west coast species Psolus chitinoides, we assumed them to be from the locally abundant species $P$. fabricii. Furthermore, their red pigmentation matched the colour of adult $P$. fabricii.

As part of a population study, we began sequencing a portion of the $16 \mathrm{~S}$ ribosomal RNA gene from adult $P$. fabricii and the collected larvae. To our surprise, the nucleotide sequence of the larvae differed from that of the adult $P$. fabricii by 17 per cent over 389 base pairs (see figure), which is a substantially greater difference than is usually observed between conspecific echinoderms ${ }^{4}$. Further work showed that the larval sequence matched that of adult $C$. frondosa exactly. In addition, the genetic analysis told us that $P$. fabricii larvae were also present in at least one of the plankton samples, though we were not able to recognize them by morphology alone. Using the sequence differences between these two species, we are now in a position to create genetic probes that can be used to identify hundreds of larvae at a time.

The use of the polymerase chain reaction for identifying marine invertebrate larvae opens up a wide range of possibilities for field studies of dispersal and recruitment. For example, isotopic studies of deep-sea gastropod shells have shown that their larvae must ascend to the near-surface waters 5,6 , but no one has ever collected a larva from this region that could be shown conclusively to be a deep-sea species. With the establishment 